\title{
Exercise-Induced Left Bundle Branch Block and Chest Pain in the Absence of Coronary Artery Disease: A Case Report and Review of the Literature
}

\author{
Keerthana Karumbaiah ${ }^{\mathrm{a}}$, Bassam Omar ${ }^{\mathrm{a}, \mathrm{b}}$
}

\begin{abstract}
Exercise-induced left bundle branch block (LBBB) is rare, but portends higher major cardiac events risk in the presence of coronary disease. It is more benign without coronary disease. We present a case of chest pain with exercise-induced LBBB with unusual features. A 59-year-old female was referred for evaluation of atypical chest pain. She was on medications for hypertension, dyslipidemia, and hypothyroidism. She denied tobacco or alcohol use. She underwent a Bruce protocol exercise treadmill test. Her resting heart rate was 76 beats per minute (BPM) and blood pressure was $150 / 85$ $\mathrm{mmHg}$. She was able to exercise for $8 \mathrm{~min}$, achieving 9 METS. Her peak heart rate was 140 BPM without ischemic ST changes. At that heart rate, she developed LBBB, and complained of her usual chest discomfort. Her peak blood pressure was 209/83 mmHg. The test was terminated, and the patient was allowed to rest. Her rhythm continued to be LBBB for $2 \mathrm{~min}$ and $30 \mathrm{sec}$ into recovery until, at a heart rate of 110 BPM, a premature ventricular contraction (PVC) resulted in resolution of LBBB. Shortly thereafter, her chest pain resolved. Subsequent cardiac catheterization revealed normal coronary arteries, ejection fraction (EF), and wall motion. Exercise-induced LBBB associated with chest pain may be a manifestation of underlying coronary disease and indicate poor prognosis. It renders the stress test uninterpretable and mandates its termination. However, certain clinical and electrocardiographic features may provide clues to non-ischemic etiology.
\end{abstract}

Keywords: Electrocardiogram; Coronary disease; Exercise testing; Left bundle branch block

\section{Introduction}

Exercise-induced left bundle branch block (LBBB) has been

\footnotetext{
Manuscript accepted for publication November 8, 2013

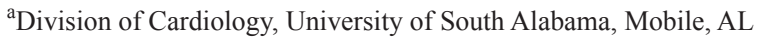
36617, USA

${ }^{\mathrm{b}}$ Corresponding author: Bassam Omar, University of South Alabama, 2451 Fillingim Street, Mobile, AL 36617, USA.

Email: Bomar@health.southalabama.edu
}

doi: http://dx.doi.org/10.4021/jmc1576w reported in approximately $0.5 \%$ of patients undergoing exercise stress testing, and it independently predicts a higher risk of death and major cardiac events [1]. Seventy percent of patients with exercise-induced LBBB were shown to have significant coronary artery disease [2]. Patients with exerciseinduced LBBB without coronary artery disease have a good prognosis compared to patients with coronary artery disease, despite frequent development of permanent LBBB [3].

\section{Case Report}

A 59-year-old Caucasian female was referred for exercise treadmill test for evaluation of atypical chest pain. She described abrupt onset of retrosternal, non-radiating sharp chest pain that developed while she was walking, and continued without increase in intensity throughout the day while active. The pain finally abated after she went to sleep. She reported history of palpitations, but no other associated symptoms. The patient had a past medical history of dyslipidemia, hypertension, hypothyroidism, and metabolic syndrome. Her medications included aspirin $81 \mathrm{mg}$ daily, gemfibrozil 600 $\mathrm{mg}$ twice daily, vytorin (ezetimibe/simvastatin) $10 / 10 \mathrm{mg}$ at bedtime, levothyroxine $112 \mathrm{mcg}$ daily, and lisinopril $10 \mathrm{mg}$ daily. She had family history of dyslipidemia, hypertension, and diabetes mellitus, but no premature coronary artery disease or sudden cardiac death. She denied tobacco or alcohol use; she was a retired school teacher. Her vital signs revealed heart rate 72 beats per minute (BPM), regular, blood pressure 142/80 mmHg, weight of $207 \mathrm{lbs}$, and height 5' 5'. Physical examination did not reveal any cardiac or pulmonary abnormalities. ECG revealed sinus rhythm at $72 \mathrm{BPM}$, otherwise normal (Fig. 1).

The patient underwent a Bruce protocol exercise treadmill test. She was able to exercise for $8 \mathrm{~min}$, achieving 9 METS. Her peak heart rate was 140 BPM ( $87 \%$ of maximal predicted heart rate) without ischemic ST changes, at which rate she developed LBBB, and complained of her usual chest discomfort (Fig. 2). The test was terminated, and the patient was allowed to rest. Her rhythm continued to be LBBB until, at a heart rate of $110 \mathrm{BPM}$, a premature ventricular contraction (PVC) resulted in a compensatory pause and resolution 


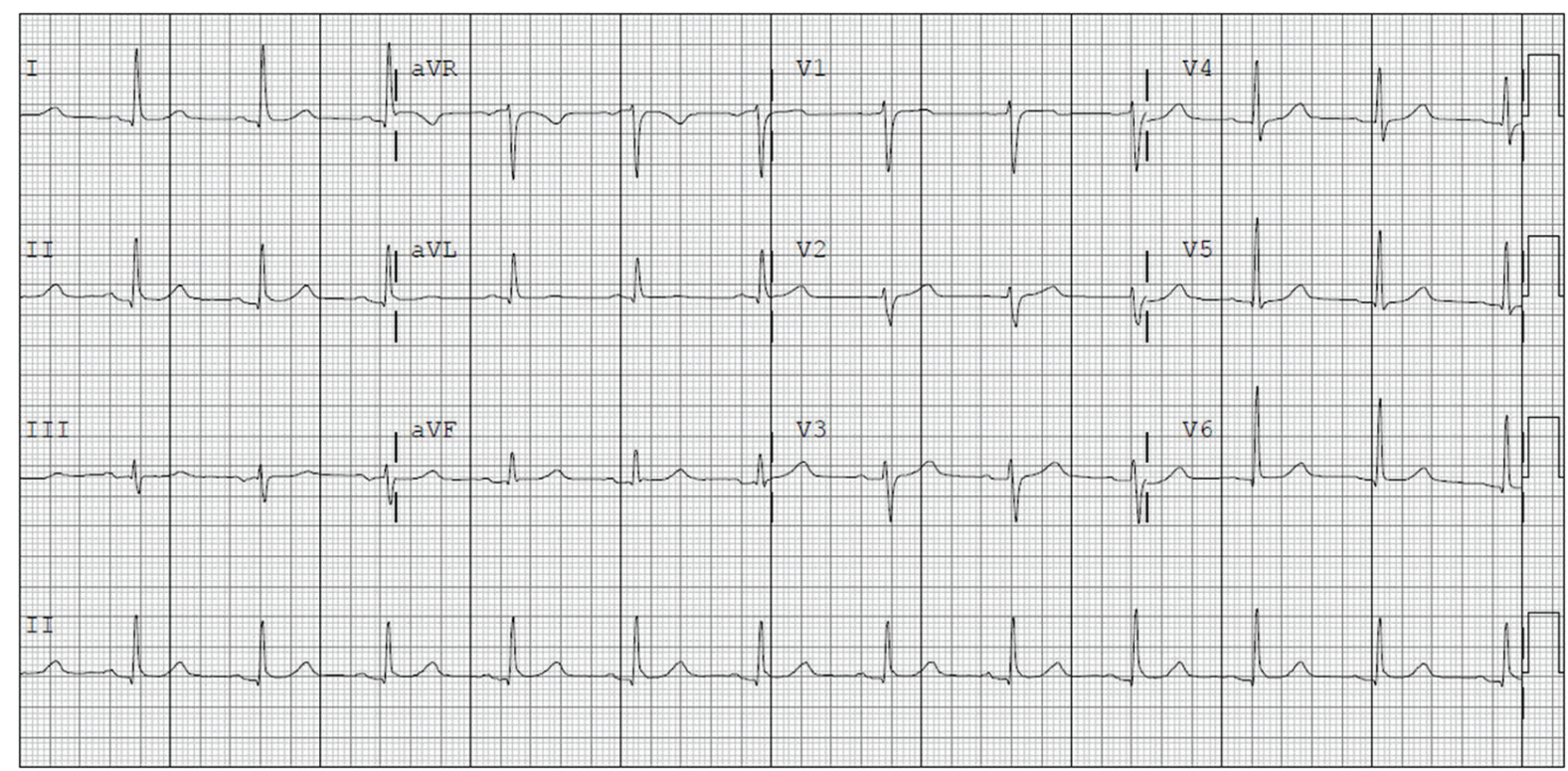

Figure 1. Baseline electrocardiogram obtained prior to exercise treadmill test.

of the LBBB (Fig. 3). Shortly thereafter, her chest pain resolved. The patient underwent cardiac catheterization which revealed an ejection fraction of 55\%, no wall motion abnormalities, and angiographically normal coronary arteries.

\section{Discussion}

The association of exercise-induced LBBB and chest pain has been previously reported [4]. Virtanen et al [5] reported a triad of exertional chest discomfort, transient rate-dependent LBBB, and normal coronary arteries in patients with clinical symptoms resembling effort angina. They, however, noted that the symptom onset was abrupt (simultaneous with the appearance of LBBB); the pain was localized, non-radiating; and that palpitation and "walk through" phenomenon were often present. Our patient's chest pain shared those features, which were reproduced during the stress test, as her pain

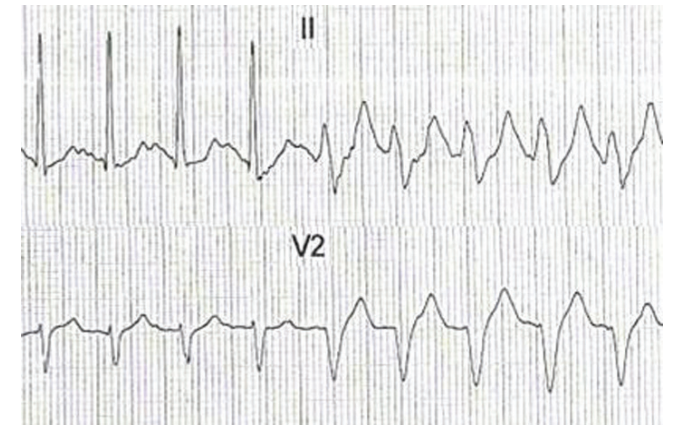

Figure 2. Electrocardiographic tracing at peak exercise, with development of rate-related LBBB at 140 BPM. was localized, abrupt, coinciding with the development of LBBB, and resolved after resolution of the LBBB. The heart rate at which the LBBB occurred in our patient was 140 BPM, which also suggests a non-ischemic etiology. Vasey et al [6] found no coronary disease in patients who developed exercise-induced LBBB at a heart rate of 125 BPM or higher, while the incidence of coronary artery disease was $50 \%$ when the LBBB developed at a heart rate less than 125 BPM.

It is interesting to note that our patient had no chest pain while her heart rate was 140 BPM before developing LBBB. However, after developing LBBB, her chest pain persisted despite the decrease in the heart rate to $110 \mathrm{BPM}$, until the LBBB resolved. This indicates that her chest pain was related to the development of LBBB, rather than an increase in heart rate or ischemia. Issa et al [7] explained that during slowing of the heart rate, rate-related intraventricular con-

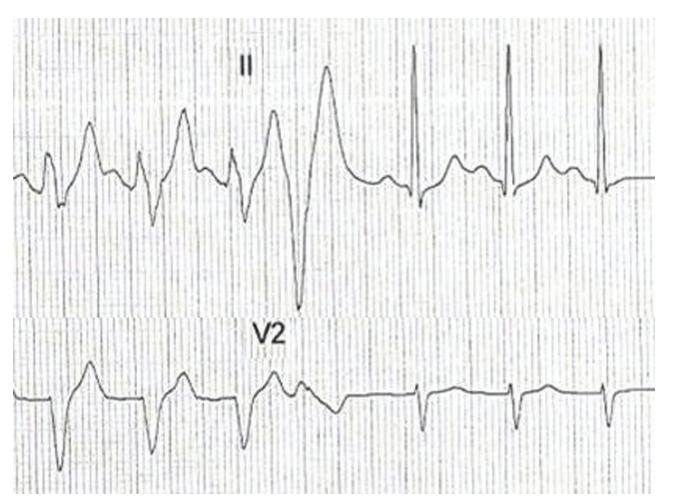

Figure 3. Electrocardiographic tracing $2 \mathrm{~min}$ and $30 \mathrm{sec}$ into recovery, with resolution of rate-related LBBB at a heart rate of 110 BPM, following a PVC. 
duction often fails to normalize at the critical cycle length which initiated the aberration. Therefore, the heart rate has to slow down more than would be expected to reestablish normal conduction. In our patient, a compensatory pause following a PVC apparently provided the critical cycle length necessary for normal conduction. The absence of ischemic ECG changes before the development of LBBB during exercise, and after the resolution of the LBBB at rest, provides further clue that her chest pain and LBBB were unlikely to be ischemic.

Little is known about the mechanism of chest pain in exercise-induced LBBB. One explanation is that abrupt ventricular asynergy as a result of altered electrical conduction may be perceived as chest pain. Virtanen et al [5] performed kinetocardiographic recordings which showed sudden paradoxical cardiac motion at the onset of LBBB, followed by chest pain. Normal cardiac motion was restored with normal conduction. Mora et al [8] found increased lactate concentration in the coronary sinus of a patient with exercise-induced LBBB, which may be indicative of localized coronary insufficiency. Ozcan et al [9] reported exercise-induced LBBB associated with slow coronary flow in a patient complaining of exertional angina.

There is no specific treatment available for chest pain caused by rate-related LBBB. Heinsimer et al [10] reported a 47-year-old female with chest pain associated with raterelated LBBB whose heart rate at onset of LBBB, after 3 months of exercise training, rose from 133 to 175 BPM. She no longer developed symptoms during routine daily activities or exercise. Shenoy and Sattur [11] reported a 48-yearold man with chest pain associated with rate-related LBBB whose symptoms resolved after starting a low-dose non-selective beta-blocker. Tanaka et al [12] reported a 53-year-old male with non-ischemic cardiomyopathy (ejection fraction (EF) 35\%) and exercise-induced LBBB at 100 BPM; after 5 months of carvedilol and candesartan therapy for CHF, his EF improved to $49 \%$, and the heart rate at which LBBB was induced increased to $126 \mathrm{BPM}$.

\section{Conclusion}

Exercise-induced LBBB is rare, and while it independently predicts a higher risk of death and major cardiac events, especially in patients with underlying significant coronary artery disease, it appears to be associated with good prognosis in the absence of significant coronary disease. It renders the ECG uninterpretable for ischemia, and thereby mandates termination of the stress test, especially in the presence of chest pain. There are several clues presented which may point towards a non-ischemic etiology. Further research is needed to clarify the mechanism of this condition, and investigate potential therapies.

\section{References}

1. Grady TA, Chiu AC, Snader CE, Marwick TH, Thomas JD, Pashkow FJ, Lauer MS. Prognostic significance of exercise-induced left bundle-branch block. JAMA. 1998;279(2):153-156.

2. Williams MA, Esterbrooks DJ, Nair CK, Sailors MM, Sketch MH. Clinical significance of exercise-induced bundle branch block. Am J Cardiol. 1988;61(4):346348.

3. Candell Riera J, Oller Martinez G, Vega J, Gordillo E, Ferreira I, Pena C, Castell J, et al. [Exercise-induced left bundle-branch block in patients with coronary artery disease versus patients with normal coronary arteries]. Rev Esp Cardiol. 2002;55(5):474-480.

4. Ninan M, Swan J. Can left bundle branch block cause chest pain? Br J Cardiol. 2002;9:230-232.

5. Virtanen KS, Heikkila J, Kala R, Siltanen P. Chest pain and rate-dependent left bundle branch block in patients with normal coronary arteriograms. Chest. 1982;81(3):326-331.

6. Vasey C, O’Donnell J, Morris S, McHenry P. Exerciseinduced left bundle branch block and its relation to coronary artery disease. Am J Cardiol. 1985;56(13):892-895.

7. Issa Z, Miller J, Zipes D. Intraventricular Conduction Abnormalities. In: Clinical Arrhythmology and Electrophysiology: A Companion to Braunwald's Heart Disease. 2nd ed. Philadelphia: Elsevier Saunders; 2012. p. 194-211.

8. Mora B, Douard H, Barat JL, Broustet JP. [Simultaneous occurrence of left heart block and chest pain during exertion]. Arch Mal Coeur Vaiss. 1987;80(12):1807-1811.

9. Ozcan F, Maden O, Ozlu M, et al. Exercise induced left bundle branch block in a patient with slow coronary flow. Abant Med J. 2012;1(3):156-158.

10. Heinsimer JA, Skelton TN, Califf RM. Rate-related left bundle branch block with chest pain and normal coronary arteriograms treated by exercise training. Am J Med Sci. 1986;292(5):317-319.

11. Shenoy C, Sattur S. The heart remembers. Am J Med. 2006;119(10):837-838.

12. Tanaka H, Hiraishi M, Miyoshi T, Tsuji T, Kaneko A, Ryo K, Yamawaki K, et al. Exercise-induced left bundle branch block and subsequent mechanical left ventricular dyssynchrony--resolved with pharmacological therapy. Cardiovasc Ultrasound. 2011;9(1):4. 